This is the author's final, peer-reviewed manuscript as accepted for publication. The publisher-formatted version may be available through the publisher's web site or your institution's library.

\title{
Impulsive choice behavior in four strains of rats: evaluation of possible models of Attention Deficit/Hyperactivity Disorder
}

Ana Garcia and Kimberly Kirkpatrick

How to cite this manuscript

If you make reference to this version of the manuscript, use the following information:

Garcia, A., \& Kirkpatrick, K. (2013). Impulsive choice behavior in four strains of rats: Evaluation of possible models of Attention Deficit/Hyperactivity Disorder. Retrieved from http://krex.ksu.edu

\section{Published Version Information}

Citation: Garcia, A., \& Kirkpatrick, K. (2013). Impulsive choice behavior in four strains of rats: Evaluation of possible models of Attention Deficit/Hyperactivity Disorder. Behavioural Brain Research, 238(1), 10-22.

Copyright: @ 2012 Elsevier B.V.

Digital Object Identifier (DOI): doi:10.1016/j.bbr.2012.10.017

Publisher's Link:

http://www.sciencedirect.com/science/article/pii/S016643281200664X

This item was retrieved from the K-State Research Exchange (K-REx), the institutional repository of Kansas State University. K-REx is available at http://krex.ksu.edu 
Impulsive choice behavior in four strains of rats:

Evaluation of possible models of Attention Deficit/Hyperactivity Disorder

Ana Garcia and Kimberly Kirkpatrick

Kansas State University

Corresponding author: Kimberly Kirkpatrick, Department of Psychology, 492 Bluemont Hall, 1100 Mid-Campus Drive, Kansas State University, Manhattan, KS 66506-5302. E-mail: kirkpatr@ksu.edu. Tel: 785-532-0805. Fax: 785-532-5401. 


\begin{abstract}
Several studies have examined impulsive choice behavior in spontaneously hypertensive rats (SHRs) as a possible pre-clinical model for Attention-Deficit/Hyperactivity Disorder (ADHD). However, this strain was not specifically selected for the traits of ADHD and as a result their appropriateness as a model has been questioned. The present study investigated whether SHRs would exhibit impulsive behavior in comparison to their control strain, Wistar Kyoto (WKY) rats. In addition, we evaluated a strain that has previously shown high levels of impulsive choice, the Lewis (LEW) rats and compared them with their source strain, Wistar (WIS) rats. In the first phase, rats could choose between a Smaller-sooner (SS) reward of 1 pellet after $10 \mathrm{~s}$ and a Larger-later (LL) reward of 2 pellets after $30 \mathrm{~s}$. Subsequently, the rats were exposed to increases in LL reward magnitude and SS delay. These manipulations were designed to assess sensitivity to magnitude and delay within the choice task to parse out possible differences in using the strains as models of specific deficits associated with ADHD. The SHR and WKY strains did not differ in their choice behavior under either delay or magnitude manipulations. In comparison to WIS, LEW showed deficits in choice behavior in the delay manipulation, and to a lesser extent in the magnitude manipulation. An examination of individual differences indicated that the SHR strain may not be sufficiently homogeneous in their impulsive choice behavior to be considered as a viable model for impulse control disorders such as ADHD. The LEW strain may be worthy of further consideration for their suitability as an animal model.

Keywords: impulsive choice; impulse control; differences among rat strains; attention deficit/hyperactivity disorder; individual differences
\end{abstract}




\section{Introduction}

Attention-Deficit/Hyperactivity Disorder (ADHD) is a developmental neuropsychiatric disorder that is estimated to affect between $2 \%$ and $12 \%$ of school-aged children, and approximately $4 \%$ of adults [1-4]. ADHD is characterized by a cross-situational pattern of inattention, hyperactivity, and/or impulsivity that interferes with appropriate social and/or academic functioning [5]. There are three subtypes of ADHD: predominantly inattentive subtype (ADHDIA), predominantly hyperactive-impulsive subtype (ADHD-HI), and combined subtype (ADHDC). In addition to the subtypes of ADHD, several endophenotypes based on quantitative psychological deficits have been proposed including: shortened reward delay gradients, and impairments in response inhibition/executive functioning, temporal processing, working memory and reaction time variability [6]. As a result, it has been proposed that ADHD is a heterogeneous condition [7-9], and that there are multiple potential causal pathways to ADHD [10-13].

One factor that has been examined in ADHD patients is the increased prevalence of impulsive choice behavior [e.g., 7, 9]. Impulsivity is a multi-faceted construct that includes both cognitive (i.e., impulsive choice) and motor (i.e., impulsive action) components [14, 15]. Impulsive choice tasks involve the presentation of choices between smaller rewards that are available sooner (SS rewards) versus larger rewards available later in the future [LL rewards; 16]. Choice of SS over LL rewards that result in lower reward-earning rates is commonly termed impulsive, whereas the opposite behavior is termed self-controlled [17, 18].

Impulsive choice behavior has been linked with a variety of behavioral problems including increased risk of drug and alcohol abuse [see 19, 20, 21 for recent reviews], gambling [21-25] and poor financial decision making [26]; impulsive choice also predicts drug relapse following treatment [27-29]. ADHD is a vulnerability disorder for drug addiction, perhaps due 
to increased impulsive choice behavior [30,31]. Impulsive choice behavior has been proposed to arise from the underlying process of temporal discounting. Temporal discounting refers to the decline in reward value as a function of delay until receipt of reward.

With regard to ADHD, children and adults expressing ADHD symptoms are more likely to select the SS choice, even when choosing that option may be less profitable, indicating increased impulsive choice behavior [32-44], however Scheres et al. [45] did not report any differences in impulsive choice between an ADHD sample and controls. They note that the failure to replicate may have been due to the relatively small sample size coupled with the use of a novel procedure with varying delays and amounts that may not have been sensitive enough to detect differences. The increased selection of the SS outcome has been interpreted as due to increased impulsive choice attributable to steeper temporal discounting [46, 47], escape from delay due to the overall trial length [delay aversion, 48, 49], or a combination of both processes $[50,51]$. A shortened delay gradient has been proposed as a core deficit in ADHD and a candidate endophenotype for the disorder $[6,11]$ that is proposed to emerge early and potentially lead to other symptom development in $\operatorname{ADHD}[11,47]$.

Impulsive choice tasks require processing of both temporal and reward amount information, and deficits in either of these processes could account for different patterns of impulsive choice behavior. As a result, two individuals could demonstrate impulsive choice behavior, but for different reasons due to deficits in the two underlying systems.

An increasing body of research has suggested that several characteristics of temporal information processing (e.g., duration discrimination, duration reproduction, finger tapping) may be impaired in ADHD [see 52 for a review]. ADHD is also associated with deficits in timing accuracy and precision [53-60], and one effect of methylphenidate, a common treatment for 
$\mathrm{ADHD}$, is to increase timing precision [55]. ADHD is associated with abnormalities in the nigrostriatal dopamine system [61], which has also been implicated as the source of the intervaltiming clock system [e.g., 62, 63], so it is possible that impulsive choice behavior in ADHD could emerge from deficits in temporal processing.

Impairments in reward processing are also associated with ADHD [33, 61, 64-66]. ADHD is characterized by deficits in the mesolimbic dopamine system [11, 65, 67-73], which is associated with reward anticipation and incentive motivation [74-78]. Strohle et al. [71] demonstrated a direct link between nucleus accumbens (a key structure in the mesolimbic pathway) activation and anticipation of gains during an impulsive choice task, with ADHD patients, demonstrating lower activation and increased impulsive choices.

Given the link between ADHD and impulsive choice, and the importance of impulsive choice in other serious behavioral problems such as drug abuse and gambling, it is pertinent to develop robust pre-clinical models for testing new interventions for ADHD and to pinpoint the possible neural mechanisms of ADHD. One of the most widely tested models is the spontaneously hypertensive rat (SHR), which were created through selective breeding to have high blood pressure recordings. Besides hypertension, the selection also produced increased activity, impulsivity, deficits in sustained attention, and alterations in the dopaminergic system, all of which are also characteristics of ADHD [79, 80], and as a result the SHR has been advanced as the best-validated animal model of ADHD [81]. In relation to impulsive choice, SHRs choose fewer LL rewards than WKY rats [82-84], and have a shorter mean adjusting delay [85], indicating a steeper delay of reinforcement gradient [see also 66, 86]. However, other research has indicated no differences in impulsive choice behavior between SHR and WKY strains [87], and the lack of steeper discounting functions in SHRs was also reported in the 
successive-encounters procedure, an operant simulation of natural foraging [88]. The discrepancy in the literature could be due to the use of different procedures which may not be equally sensitive to detect differences between strains, or could indicate that impulsive choice behavior is not a robust phenotype of the SHR strain.

An additional issue with the SHR strain is that they have not been thoroughly evaluated in relation to temporal and/or reward processing deficits within the context of an impulsive choice task. Given that both deficits are associated with ADHD, it is critical to determine whether the SHR strain exhibits impairments in one or both of these processes to evaluate their suitability as an animal model for different sub-populations of ADHD. Further evaluation of the SHR strain will also provide accumulating evidence of whether the deficits in SHRs are a robust phenotype of the strain given the previous inconsistencies in the literature.

A second possible animal model was also evaluated, the Lewis (LEW) strain. LEW rats have not been evaluated as an animal model of ADHD, but this strain has shown to make more impulsive choices in comparison to Fischer 344 rats [89-94]. Such differences in choice are important as they may provide an avenue for exploring impulsive choice in a new potential model of ADHD.

Accordingly, the present research examined impulsive choice behavior in two potential impulsive strains (SHR and LEW) and their source strains (WKY and WIS) to assess these two strains as potential models of ADHD. The strains were chosen because SHR and LEW have been previously reported to be impulsive, the WKY are the source strain and also the most common control strain for the SHRs, and the WIS strain is the source strain for the LEW. Note that the goal of this study was to compare the two potentially impulsive strains to their source strains rather than to make comparisons based on other factors such as neurobiological 
differences. In addition, analyses of individual differences were conducted to assess the relative homogeneity within the impulsive and control strains to determine whether the impulsive strains could serve as a model for specific symptoms of ADHD.

Separate manipulations of SS delay and LL magnitude were delivered to all rats; this approach has been previously used to disentangle the role of temporal and reward processing in impulsive choice $[78,95]$. If the impulsive strains display deficits in only one of the manipulations, then this would indicate that the strain could be evaluated more thoroughly for specific temporal and/or reward processing deficits related to impulsive choice behavior in ADHD.

\section{Materials and Methods}

\subsection{Animals}

The animals were 36 experimentally-naive male rats from four different strains $(n=9)$ : SHR (SHR/NCrl), WKY (WKY/NCrl), WIS (WIS/Crl), and LEW (LEW/Crl) from Charles River Inc. (Wilmington, MA, USA). The rats were approximately 60 days old at the beginning of the experiment. The rats were housed in pairs in plastic shoe box cages and were handled daily. After habituation to the conditions of the animal colony, body weights were reduced to approximately $85 \%$ of free-feeding weight by restricted feeding consisting of the food pellets earned in a session plus an additional ration of lab chow in the home cage. Water was available ad libitum in the home cage and experimental chamber. Lights were on a 12:12 hr reversed light-dark cycle with lights on at 8 p.m.

\subsection{Apparatus}

All phases of the experiment were conducted in a set of 18 operant chambers (Med Associates, Vermont, USA). Each chamber $(25 \times 30 \times 30 \mathrm{~cm})$ was enclosed in a ventilated, 
sound-attenuating cubicle $(74 \times 38 \times 60 \mathrm{~cm})$. The floor of the chamber was a stainless steel grid comprised of nineteen 0.5-cm diameter bars (Model ENV-005). Each chamber had two retractable response levers $(\mathrm{ENV}-112 \mathrm{CM})$ located $2.1 \mathrm{~cm}$ above the floor in the front wall; each lever was $4.8 \mathrm{~cm}$ wide. A $5.1 \times 5.1 \mathrm{~cm}$ pellet receptacle $(\mathrm{ENV}-200 \mathrm{R} 2 \mathrm{M})$ was located in the center of the front wall, $2.5 \mathrm{~cm}$ above the floor, and this received 45-mg Noyes precision food pellets (Research Diets, New Brunswick, NJ) from a modular magazine pellet dispenser (MED Associates, Model ENV 203M). The chambers were located in two separate rooms, with six chambers in one room and twelve chambers in the other room. The presentation of stimuli and the collection of data were controlled by Dell personal computers using the Medstate programming language (Med-PC-IV, MED Associates).

\subsection{Procedure}

2.3.1. Pre-training. In the initial session, all rats received magazine training with single food pellets delivered on a variable time 60 -s schedule for $1 \mathrm{hr}$. The following two sessions consisted of continuous reinforcement training, with a single food pellet delivered for each lever press on both the left and the right levers, one lever per day (order counterbalanced), for a total of 30 lever presses. Each session lasted a maximum of $2 \mathrm{hr}$. Most of the rats started pressing the levers with this procedure; rats that did not press the levers were given hand shaping until they began pressing. During the following two sessions, both left and right levers were trained simultaneously in six blocks within a session, each block consisted of 20 food deliveries per lever. In the first two blocks, lever pressing was reinforced according to a fixed ratio 1 schedule. The next two blocks followed a random ratio schedule with a mean of three lever presses per food delivery and the last two sessions followed a random ratio schedule with a mean of five 
lever presses per food delivery. Sessions finished when the rats received 120 total food deliveries.

2.3.2. SSLL training. Sessions were composed of forced choice, free choice, and probe trials. Forced choice trials involved insertion of one of the levers. An initial response on that lever resulted in onset of the cue light above the lever. After the target fixed interval elapsed, the first response on the lever resulted in lever retraction and food delivery. Probe trials involved insertion of only one of the levers, lasted for $90 \mathrm{~s}$ and were non-reinforced. Lever presses were monitored to assess the pattern and rate of responding in the absence of reinforcement, but had no consequence [see 96]. Free choice trials were initiated by inserting both levers. Following a choice response, the alternative lever was withdrawn, the cue light above the chosen lever was turned on and a fixed interval schedule was initiated on the chosen lever. Once the target interval elapsed, the next response on the lever resulted in lever retraction and food delivery. In the first phase, SS trials resulted in delivery of 1 pellet (contingent on a lever press following the fixed interval) after a 10-s delay, whereas LL trials resulted in the delivery of 2 pellets after a 30s delay. All trials were separated by a fixed 60 -s ITI. A fixed ITI more closely mimics real-life choice situations because it allows for reward maximization [97]. In the present study, in all conditions, SS choices were costly because they reduced total rewards earned so this produced a situation where SS choices were maladaptive, especially when the LL reward magnitude was larger or the SS delay was longer. The sessions were conducted during the dark phase of the light:dark cycle and consisted of two blocks, each containing 8 SS forced, 2 SS probe, 8 LL forced, $2 \mathrm{LL}$ probe, and 30 free choice trials. Sessions lasted for $2.5 \mathrm{hr}$, and water was freely available in the chambers throughout the session. 
Following the baseline procedure with the 10-s, 1-pellet SS vs. the 30-s, 2-pellets LL choices, additional training occurred with LL magnitude and SS delay increases in a counterbalanced order. In each strain approximately half of the rats ( 5 in one sub-group and 4 in the other) received the LL magnitude increase first, followed by a return to the baseline condition and then followed by the SS delay increase task. The remaining rats received the SS delay increase task first, followed by a return to baseline and then the LL magnitude increase task. In the LL magnitude task, the LL reward was increased to 3 pellets, and then later to 4 pellets in separate phases. The SS delay task consisted of an increase in the SS delay to $15 \mathrm{~s}$, and then later to $20 \mathrm{~s}$ in separate phases. The return to the baseline condition between successive tasks involved the original training conditions (10 s, 1 pellet vs. $30 \mathrm{~s}, 2$ pellets), but the SS and LL lever assignments were switched to remove any biases that may have emerged in the initial task. Training in each phase lasted for 20 sessions, except for the second baseline phase which lasted for 30 sessions.

\subsection{Data analysis}

All analyses were conducted in SPSS, using the General Linear Model routine, on the last five sessions of each phase, when choice behavior was stable. For all of the ANOVAs, separate analyses were conducted for the WKY vs. SHR and the WIS vs. LEW as these pairs of strains were coupled according to their genetic relationship. An additional analysis of the SHR vs. WIS was also conducted to check for differences between the SHRs and their original source strain.

\subsubsection{Choice analysis.}

2.4.1.1. Percentage of $L L$ choices. The data analysis focused on the free choice trials, where both the SS and LL options were present. The probe trials were subjected to thorough data analyses, which are reported in Garcia Aguirre's [98] thesis. Because the primary focus of 
the present paper was on choice behavior, these analyses are not reported here. The probability of accepting the LL choice for each phase was obtained from the free choice trials and was computed by dividing the number of LL choices by the total number of choices available (30 per session) and multiplying by 100 .

To gain an index of the strength of the contribution of the manipulated variables (e.g., the strains) in comparison to individual differences within a strain, two measures of effect size were computed. The first measure was a standard $\eta^{2}$ statistic, which was used to determine the percentage of variance accounted for by each of the variables in the experiment: $\eta^{2}=\frac{S S Q_{E \text { Effect }}}{S S Q_{\text {Total }}}$, where SSQEffect was the sum of squares associated with each effect in the model (Strain, Phase, or Session) and $S S Q_{\text {Total }}$ was the total sum of squares adjusted for the grand mean. To compute the variance accounted for by the inter-individual differences, $S S Q_{E f f e c t}$ was the error sum of squares from the Strain effect (the same as $S S Q_{K}$ below). This is the normal error term for a betweensubjects effect computation, reflecting the inter-individual differences within groups. All of the sums of squares were obtained from the General Linear Model output in SPSS. The $\eta^{2}$ values add to $100 \%$ if computations are made for all variables and error terms in the ANOVA.

The second estimate of effect size was the generalized eta-squared statistic, $\hat{\eta}_{G}^{2}[99,100]$, which was derived to deal with some of the common problems with the standard and partial $\eta^{2}$ statistics. The generalized eta-squared statistic was determined by the equation $\hat{\eta}_{G}^{2}=\frac{S S Q_{\text {Effect }}}{\delta * S S Q_{\text {Effect }}+\sum_{\text {Meas }} S S Q_{\text {Meas }}+\sum_{K} S S Q_{K}}$, where $S S Q_{E f f e c t}$ was the sum of squares associated with each effect, $S S Q_{\text {Meas }}$ includes all sources of error variance that involve measured variables 
(Strain, Phase, Session, and their interactions) and $S S Q_{K}$ is the inter-individual differences. The parameter $\delta$ was set to 1 when the effect of interest was a manipulated factor (Strain, Phase, or Session). For measurement of the effect size for the inter-individual differences, $S S Q_{E f f e c t}$ was the error sum of squares from the Strain effect $\left(S S Q_{K}\right)$. This is the normal error term for a between-subjects effect computation, reflecting the inter-individual differences within groups. In this case, $\delta$ was set to zero so that the individual differences $\left(S S Q_{K}\right)$ only contributed to the denominator once.

As an additional index of the contribution of the inter-individual differences to the experiment, an F-statistic was computed by dividing the mean square error for the Strain effect (the inter-individual differences) by the mean square error for the Session effect (the intraindividual error). Both of these error terms were obtained from the General Linear Model output in SPSS.

2.4.1.2. Log-odds ratios. Due to issues with violations of ANOVA assumptions when using proportion data, a log-odds ratio was computed for each rat's choice behavior in each phase of the delay and magnitude manipulations to attempt to minimize the impact on the statistical analyses. The log-odds ratio was determined by: $\log _{10}\left(\mathrm{~N}_{\mathrm{LL}} / \mathrm{N}_{\mathrm{SS}}\right)$ where $\mathrm{N}_{\mathrm{LL}}$ refers to the number of LL choices and $\mathrm{N}_{\mathrm{SS}}$ refers to the number of SS choices, accumulated over the last 5 sessions of each phase.

2.4.1.3. Area under the curve. The area under the curve (AUC) has been used to avoid potential problems created by the lack of consensus regarding the mathematical form of discounting functions (i.e., exponential vs. hyperbolic). The AUC is a theoretically neutral measure of discounting which requires no assumptions regarding the mathematical form of the discounting function and that provides an index of overall bias to choose the SS or LL. The 
AUC was determined by summing the area of successive trapezoids between the percentage of choices for each of the phases $[101,102]$. Given that the units between the different phases were evenly spaced, AUC was equal to $(\mathrm{y} 1+\mathrm{y} 2) / 2+(\mathrm{y} 3+\mathrm{y} 2) / 2$, where $y 1$, $\mathrm{y} 2$ and $y 3$ were the percentage of LL choices in the first, second and third phase, respectively, for the magnitude and delay manipulations. The AUCs were then divided by the maximum possible area (200) so that the values ranged from 0 to 1 . Because the AUC values are expressed in normalized units obtained from choice data, they should not be compared directly to AUC values obtained from indifference points in adjusting-amount or adjusting-delay procedures.

2.4.1.4. Slope. The slope of the choice function provides a measure of sensitivity to changes in LL magnitude or SS delay. This was computed by taking the slope of the function relating the percentage of LL choices and the LL magnitude or SS delay for each rat. Therefore, this represents the change in percentage of LL choices due to a one-unit change in LL magnitude (e.g., 2 to 3 pellets or 3 to 4 pellets) or SS delay. The slope could range from 0 to 50 .

2.4.1.5. Box plot generation. To effectively display the individual differences in choice, as well as providing central tendency and distributional information, box plots were generated for displaying the percentage of LL choices. The central line in each box plot represents the median, whereas the lower and upper halves of the box are the $25_{\text {th }}$ and $75_{\text {th }}$ percentiles, respectively. The whiskers on the box are the minimum and maximum scores for the group.

\section{Results}

\subsection{Percentage of $L L$ choices}

3.1.1. WKY vs. SHR strains. Figure 1 displays the results of the LL reward magnitude (left column) and SS delay (right column) manipulations conducted on the WKY and SHR strains. The top row displays the mean percentage of LL choices for the two strains, whereas the 
middle and bottom rows display the box plots for each individual strain (Section 2.4.1.5), with the mean overlaid on the box plot. It is apparent in examining the figure that both strains adjusted their choice behavior by increasing LL choices as the LL magnitude increased (left column) or the SS delay increased (right column). The WKY strain appeared to show slightly higher LL choices in the LL magnitude manipulation, but the two groups were virtually identical in their performance in the SS delay manipulation. It also appears from the box plots that there were considerable individual differences in choice behavior within both strains of rats.

To confirm the general patterns in the data, separate ANOVAs were conducted on the magnitude and delay tasks. Specifically, a 2 × 3 x 5 mixed analysis of variance (ANOVA) with the variables of Strain (between-groups factor), Phase (within-subjects factor) and Session (within-subjects factor) was conducted for each task. For the magnitude manipulation, there was a significant effect of LL Magnitude, $F(2,32)=47.1, p<.001, \eta^{2}=.41, \hat{\eta}_{G}^{2}=.43$, on the percentage of LL choices. An additional computation was performed to assess the contribution of the inter-individual differences (see Section 2.4.1.1). This revealed a significant contribution of Individual differences, $F(16,64)=120.3, p<.001, \eta^{2}=.37, \hat{\eta}_{G}^{2}=.68$, to the percentage of LL choices. For the delay manipulation, there was an effect of SS delay, $F(2,32)=36.4, p<.001$, $\eta^{2}=.30, \hat{\eta}_{G}^{2}=.30$, and a significant contribution of Individual differences, $F(16,64)=129.5, p<$ $.001, \eta^{2}=.52, \hat{\eta}_{G}^{2}=.75$. There were no significant effects of Strain, Session, or any interactions of these variables with other variables in the analysis.

An additional comparison was conducted on the baseline phases of the magnitude and delay manipulations to determine whether the original baseline performance differed from the subsequent baseline performance as an index of possible carry-over effects. A mixed ANOVA 
was conducted with the variables of Strain (WKY vs. SHR) and Baseline phase (magnitude vs. delay). This did not reveal any significant effects of Baseline phase, Strain, or their interaction.

3.1.2. WIS vs. LEW strains. Figure 2 displays the percentage of LL choices for the WIS and LEW strains during the LL reward magnitude (left column) and SS delay manipulations (right column). The top row displays the group means and the middle and bottom rows show the box plots for each individual strain. Both strains showed sensitivity to the change in LL reward magnitude and SS delay by increasing their LL choices. There was an indication in both tasks of lower LL choices in the LEW rats, and this was more pronounced in the delay manipulation. There appeared to be considerable inter-individual differences present in both strains.

To confirm the general patterns in the data, separate ANOVAs were conducted on the magnitude and delay tasks. Specifically, a 2 x 3 x 5 mixed analysis of variance with the variables of Strain (between-groups factor), Phase (within-subjects factor) and Session (withinsubjects factor) was conducted for each task. The ANOVA on the magnitude manipulation revealed significant effects of Magnitude, $F(2,32)=76.6, p<.001, \eta^{2}=.55, \hat{\eta}_{G}^{2}=.59$, Session, $F(4,64)=2.7, p=.04, \eta^{2}=.01, \hat{\eta}_{G}^{2}=.01$, and Session $\mathrm{x}$ Strain, $F(4,64)=3.0, p=.025, \eta^{2}=.01$, $\hat{\eta}_{G}^{2}=.01$. There also was a significant contribution of Individual differences, $F(16,64)=55.0, p$ $<.001, \eta^{2}=.21, \hat{\eta}_{G}^{2}=.57$. There was no significant main effect of Strain. Tukey pairwise posthoc tests on the Session x Strain interaction were conducted to assess differences in choice behavior within each strain between pairs of sessions. These indicated that the LEW rats had lower LL choices in Session 15 than Session 20, whereas the WIS rats did not display any significant differences in choice behavior from session to session. An ANOVA conducted on the delay manipulation revealed significant effects of Delay, $F(2,32)=42.1, p<.001, \eta^{2}=.30, \hat{\eta}_{G}^{2}=$ 
.33 , Delay x Strain, $F(2,32)=3.7, p=.035, \eta^{2}=.03, \hat{\eta}_{G}^{2}=.04$, and a significant contribution of Individual differences, $F(16,64)=74.3, p<.001, \eta^{2}=.42, \hat{\eta}_{G}^{2}=.71$. Tukey pairwise post-hoc tests on the Delay x Strain interaction (comparing the two strains at each delay) indicated lower LL choices in the LEW strain in the 15-s and 20-s SS delay conditions compared to the WIS strain, but no significant strain differences in the 10-s SS delay condition. There was no significant main effect of Strain, Session, or any other interaction.

An additional comparison was conducted on the baseline phases of the magnitude and delay manipulations using a mixed 2 × 2 ANOVA with the variables of Strain (between-groups factor) and baseline phase (within-subjects factor). This did not reveal any significant effects of Baseline phase, Strain, or their interaction.

\subsection{Log-odds Ratios}

3.2.1. WKY vs. SHR strains. A further analysis of the choice data was conducted on the log-odds ratios (see Section 2.4.1.2; data not shown) to determine whether there were any strain differences when the data were transformed to better conform to the assumptions of ANOVA. Mixed 2 x 3 ANOVAs were conducted on the log-odds ratios from the delay and magnitude tasks with the variables of Strain (between-groups factor) and Phase (within-subjects factor). For the magnitude manipulation, there was a significant effect of Magnitude, $F(2,32)=12.6, p<$ .001 , but no significant effect of Strain, or Magnitude x Strain. For the delay manipulation, there was a significant effect of Delay, $F(2,32)=50.1, p<.001$ but no significant effect of Strain or any interaction.

3.2.2. WIS vs. LEW strains. The analysis of the log-odds ratios for the WIS vs. LEW strains in the magnitude task (using a 2 × 3 mixed ANOVA) revealed a significant effect of 
Magnitude, $\mathrm{F}(2,32)=103.3, \mathrm{p}<.001$, and a Magnitude $\mathrm{x}$ Strain interaction, $\mathrm{F}(2,32)=4.3, \mathrm{p}<$ .05. There was no significant main effect of Strain. The Magnitude x Strain interaction was due to significantly higher LL choices in the WIS strain when the LL magnitude was 3 pellets, but there were no significant strain differences in the 2- or 4-pellet conditions, as determined by Tukey post-hoc tests comparing the two strains in each phase. For the delay manipulation, there was a significant effect of Delay, $\mathrm{F}(2,32)=57.1, \mathrm{p}<.001$, and Delay $\mathrm{x}$ Strain, $\mathrm{F}(2,32)=6.2, p<$ .01 but no significant main effect of Strain. Tukey post-hoc tests on the interaction (comparing the two strains at each delay) determined that the WIS strain had significantly higher LL choices in the 15- and 20-s SS delay conditions, but there were no significant differences in the 10-s baseline condition.

\subsection{Area Under the Curve}

A measure of the relative AUC (Section 2.4.1.3) was generated from the percentage of LL choice data in Figures 1 and 2 to give an index of overall bias to choose LL vs. SS [a generalized measure of self-control; 102].

3.3.1. WKY vs. SHR strains. The top panel of Figure 3 displays the mean $( \pm$ standard error of the mean, SEM) AUC values for the WKY and SHR strains in the magnitude (M; filled symbols) and delay (D; open symbols) manipulations. Separate one-way ANOVAs with the between-subjects variable of Strain were conducted on the magnitude and delay tasks, but these did not reveal any significant strain differences in the relative AUC values. An additional analysis was performed to assess overall biases in choice behavior using a one-sample t-test with a comparison value of 0.5 . Because the AUC values were normalized to range from 0 to 1 , a value of 0.5 would indicate an overall lack of bias (across all choice conditions in the magnitude or delay manipulations) to choose either option. This does not necessarily mean that choices in 
all phases were neutral, but rather that the overall pattern was not biased in one direction or the other. The analysis indicated that the WKY strain was significantly biased towards selfcontrolled (LL) choices in the magnitude task, $t(8)=3.2, p<.05$, but was not biased in either direction in the delay task. The SHR strain did not significantly deviate from neutrality in their overall bias.

3.3.2. WIS vs. LEW strains. The AUC for the magnitude (M; filled symbols) and delay (D; open symbols) manipulations is displayed in the bottom panel of Figure 3 for the WIS and LEW strains. One-way ANOVAs conducted separately on the magnitude and delay tasks with the between-subjects variable of Strain revealed a trend towards an effect of Strain on both tasks, Magnitude $F(1,16)=4.4, p=.052$, and Delay $F(1,16)=3.3, p=.087$. One-sample t-tests conducted on the relative AUC values to assess biases in choice revealed a significant selfcontrol bias in the WIS strain during the magnitude task, $t(8)=5.0, p<.01$. There were no other significant biases in either strain.

\subsection{Slope of the Discounting Function}

As an additional index of possible differences in discounting, the slope of the discounting function was computed for each rat in each strain during the magnitude and delay tasks (see Section 2.4.1.4). The slopes provided an index of sensitivity to the magnitude and delay manipulations.

3.4.1. WKY vs. SHR strains. The slopes are displayed in the top panel of Figure 4 for the WKY and SHR strains for the magnitude (M; filled symbols) and delay (D; open symbols) tasks. Separate one-way ANOVAs with the between-subjects variable of Strain revealed no significant strain effects in either the delay or magnitude manipulations. One-sample t-tests were performed on each strain in each task to assess sensitivity to changes in magnitude and delay by 
comparing the slopes to zero (which would indicate no change in choice behavior over changes in magnitude or delay). This revealed significant sensitivity to both magnitude and delay in both strains, all ts $(8)>4.0, p s<.01$.

3.4.2. WIS vs. LEW strains. The slopes for the WIS and LEW strains are displayed in the bottom panel of Figure 4 for the magnitude (M; filled symbols) and delay (D; open symbols) tasks. There was no clear indication of any slope differences between the strains in the magnitude task, but the slope of the delay function did appear shallower in the LEW strain. However, the slope difference did not reach statistical significance, $F(1,16)=3.6, p=.077$. One-sample t-tests on the slopes revealed good sensitivity to both delay and magnitude in both strains, all $t \mathrm{~s}(8)>5.4$, all $p \mathrm{~s}<.01$.

\subsection{Additional analysis of WIS vs. WKY and WIS vs. SHR}

Given that the use of the WKY (NCrl) has been questioned as a control strain, for other behaviors besides impulsive choice [81, 103, 104], further analyses were conducted to compare the WIS strain with the WKY and SHR strains. The WIS strain is the original source strain for both of these inbred strains. The original percentage LL choice data for the magnitude and delay tasks as well as the log-odds ratios, slope and AUC measures were subjected to ANOVAs comparing the WIS vs. WKY and WIS vs. SHR. There were no strain differences in any of these comparisons. The largest group differences were in comparing the WIS vs. SHR on the magnitude task in both the percentage LL choice, $F(1,16)=2.8, p=.12$, log-odds ratios, $F(1,16)$ $=2.7, p=.12$, and the magnitude AUC, $F(1,16)=2.9, p=.11$. All other $F \mathrm{~s}<1$ for comparisons of the strains.

\subsection{Changes in choice behavior over the session}


To examine whether there were any changes in performance across the session, and whether this differed by strain, the percentage of LL choices was determined separately for the first vs. second half of the session (over the last 5 sessions of each phase). These choice data were collapsed across the individual magnitude and delay conditions as similar patterns of change over the session occurred in all of the individual conditions.

3.6.1. WKY vs. SHR strains. The percentage of LL choices (mean \pm SEM) of the WKY and SHR strains as a function of the first vs. second half of the session is displayed in the top panel of Figure 5 for the magnitude (WKY-M and SHR-M) and delay (WKY-D and SHR-D) tasks. As seen in the figure, both strains showed increased LL choices between the first and second half of the magnitude and delay manipulations. This was confirmed by separate $2 \times 2$ mixed ANOVAs conducted on the magnitude and delay manipulations with the between-groups variable of Strain and the within-subjects variable of Time. For the magnitude task, there was a significant effect of Time, $F(1,16)=14.8, p<.01$, that was due to increased LL choices in the second half of the session, but no significant interaction of Strain $\mathrm{x}$ Time. The same pattern was observed in the analysis of the delay manipulation, with a significant effect of Time, $F(1,16)=$ $5.4, p<.05$, but no significant interaction with Strain.

3.6.2. WIS vs. LEW strains. The percentage of LL choices (mean \pm SEM) of the WIS and LEW strains as a function of the first vs. second half of the session is displayed in the bottom panel of Figure 5 for the magnitude (WIS-M and LEW-M) and delay (WIS-D and LEW-D) tasks. For the magnitude task, both strains increased their LL choices over the session. This was confirmed by a $2 \times 2$ ANOVA with the between-subjects variable of Strain and the withinsubjects variable of Time, which revealed a significant effect of Time, $F(1,16)=12.9, p<.01$, but no significant interaction with Strain. For the delay manipulation, there was a different 
pattern with the WIS strain displaying an increase in LL choices but the LEW strain decreasing their LL choices over the session. A 2 x 2 ANOVA on the delay manipulation revealed a significant Time $\mathrm{x}$ Strain interaction, $F(1,16)=5.5, p<.05$, but no significant main effect of Time. Tukey pairwise post-hoc tests comparing each strain during each half of the session revealed no significant difference in the strains during the first half of the session, but the LEW rats had significantly lower LL choices than the WIS strain in the second half of the session.

\subsection{Additional analysis of individual differences}

To gain a deeper understanding of the patterns of individual differences in overall selfcontrol (measured by AUC) versus the sensitivity to magnitude or delay changes (measured by the slope), Figure 6 displays a scatter plot of the AUC vs. slope for the magnitude (top panel) and delay (bottom panel) tasks comparing all four strains. The figure is sectioned into quartiles which represent different patterns of performance. The AUC is divided at 0.5 , because this represents overall neutrality in choice behavior; AUC values greater than 0.5 indicate a selfcontrol bias and less than 0.5 indicate an impulsive bias. The slope is divided at 25 , which is the mid-point of the possible range of slopes. A slope of 0 would indicate no change in performance, whereas a slope of 50 would indicate maximal change over the choice function. Ideal performance would fall within the upper-right quartile, which is an indicator of generalized self-control coupled with good sensitivity to change. The least ideal pattern would be the lowerleft quartile, which is generalized impulsive choice coupled with poor sensitivity to change. Individuals in this quartile are generalized SS responders and demonstrate impulsive tendencies even when the reinforcement contingencies are clearly in favor of the LL outcome [see 50 for evidence of SS responders in an ADHD sample]. In examining the patterns in the different strains, it is apparent that the WIS and WKY control strains performed most ideally in the 
magnitude task with 8 of the 9 rats in each strain displaying self-controlled choices and 5 of those 8 displaying high sensitivity to change. The SHR strain was the most widely distributed in the magnitude task, with at least 1 rat in each quartile and 2 rats in the SS responder quartile. In the delay task, the WIS strain showed the best performance with 6 of the 9 rats displaying selfcontrol coupled with high sensitivity to change. The LEW strain displayed the poorest performance with 5 of the 9 rats in the SS responder quartile. The WKY and SHR strains were distributed across the quartiles. In addition, there were more SS responder rats across all strains in the delay task.

\section{Discussion}

The purpose of the study was to compare impulsive choice behavior in SHR and LEW rats, which have previously demonstrated increased choices of the SS outcome, to their genetically-compatible control strains, the WKY and the WIS rats, respectively, on an impulsive choice task. Separate assessments were conducted for LL magnitude and SS delay increases to parse out the strains as possible models for specific symptoms of ADHD and to examine whether any deficits were general patterns of choice behavior or specific to magnitude or delay manipulations. The overall pattern of results suggests that the SHR strain is not a potential model of impulsive choice deficits that may be an endophenotype of ADHD due to excessive inter-individual differences and weak to no differences from controls. The LEW strain demonstrated some potential as a model for examining deficits in adjusting to changes in SS delays, and to a lesser extent LL magnitude, which may be a potential model for temporal (and possibly reward processing) deficits coupled with impulsive choice behaviors in ADHD. 


\subsection{WKY vs. SHR strains}

The results showed that both of the strains, WKY and SHR, were sensitive to the LL reward magnitude manipulation (left column of Figure 1). The preference for LL did not differ between the SHR and WKY rats in either the original choice data or in the log-odds ratio transformed choice data. There also were no strain differences in overall self-control, measured by the AUC, or in sensitivity to LL magnitude, measured by slope (top panel of Figures 3 and 4). And, there were no strain differences in the changes that occurred across the session; both strains increased their LL choices in both tasks over the course of a session. The increase in LL choices indicates that neither strain was displaying any satiety effects in their choice behavior over the session, but rather appeared to gravitate toward the more profitable LL option over the session. The combined results suggest that the SHR and WKY strains were highly similar in their patterns of performance over the magnitude task.

Similarly, both strains were sensitive to the SS delay manipulations (right panel of Figure 1). There also were no differences between the strains in the relative AUC or slope of the discounting function (top panel of Figures 3 and 4), and no strain differences in performance across the session (Figure 5). The combined results suggest that the SHR and WKY strains were highly similar in their patterns of performance over the delay task.

It appears that the SHR rats were not more impulsive than the WKY rats in either the magnitude or delay manipulations. The results were unlikely due to any weaknesses in the experimental methods because both strains showed systematic variations in choice as a function of LL magnitude and SS delay. In addition, there were no significant differences in baseline performance between the delay and magnitude task in either strain, indicating that there were no significant carry-over effects that may have masked strain differences. Finally, observation of 
strain differences between LEW and WIS strains (discussed below) indicates that the task was sufficiently sensitive to detect strain differences. Thus, it is possible that steeper discounting may not be a reliable or robust phenotype of the SHR strain.

The present results are consistent with the findings from Adriani, et al. [87] who reported no differences between SHR and WKY rats in an impulsive choice task; however, the results are inconsistent with other reports of preference for smaller sooner rewards in SHR rats [82-86]. One possible reason for the discrepant results may be due to the commercial supplier of the rats. All of the previous studies [82-87] used different suppliers (Charles River Italia, Centre d'Elevage René Janvier, France, Charles River USA, the Department of Laboratory Animal Since at the University of Otago, New Zealand, and Harlan USA). It is possible that subtle differences in breeding criteria may result in differences in the levels of impulsive choice exhibited by strains supplied by different breeders [see 105].

Even more concerning is the observation of high inter-individual variability within SHRs obtained from the same breeder, as reported here and by Adriani et al. [87]. They reported that when the rats of the SHR strain were considered as a whole, they did not differ from their WKY controls on their LL choice preference; but if the SHR were segmented into subpopulations, the most impulsive subpopulation did differ from the WKY controls. Our results also showed that there was high inter-individual variability present in both SHR and WKY strains. Individual differences accounted a significant proportion of the total variance and contributed considerably more variance than the strain across both tasks. In addition, the SHR strain was widely distributed in the analysis of self-control bias vs. sensitivity; the SHR strain was the only strain with rats in all four of the quartiles in this analysis in both the magnitude and delay tasks (Figure 6). A much larger sample size is required to conduct a full analysis of the distributional 
properties of the strains to validate the presence of subpopulations. Nevertheless, the presence of such large inter-individual differences would appear to present a serious challenge for the consideration of the SHR as a viable model for disordered impulsive choice in ADHD, and this factor may explain why some researchers have failed to reproduce previously published results obtained with the SHR. The SHR strain has not been specifically selected for impulsivity or other markers of $\mathrm{ADHD}$, so it is perhaps not surprising to observe such large inter-individual differences in a trait that is orthogonal to the selection criterion for breeding (hypertension).

An additional possible issue with the present results was due to the use of the WKY/NCrl strain as a control strain for the SHR/NCrl strain. The different WKY substrains have been reported to exhibit substantial behavioral and genetic differences and the WKY/NCrl substrain has been proposed as a possible animal model for the ADHD-IA subtype due to deficits in sustained attention $[81,103,104]$. However, this substrain has not been reported to suffer from any increases in impulsive choice behavior, so it is not clear whether the use of this substrain of WKY rats would have affected the present results. In addition, neither the SHR nor WKY strains differed from the outbred WIS strain, which is the original source strain for both strains, so this further suggests that there were no deficits in impulsive choice in either strain.

\subsection{WIS vs. LEW strains}

As a first approach to evaluate another potential animal model of impulsive choice deficits in ADHD, the present experiment evaluated the effects of LL reward magnitude and SS delay on choice behavior in the LEW rats, a strain that has been reported to make more impulsive choices in delay discounting tasks [89-94].

In the reward magnitude manipulation, the preference for the LL reward increased when the LL reward incremented from 2 to 3 to 4 pellets in separate phases. There was an indication 
of lower LL choices in the reward magnitude manipulation (left column of Figure 2). This did not reach significance with the original choice measure, but there was a significant deficit in LL choices at the magnitude of 3 pellets using the log-odds ratio measure. Both strains displayed an increase in LL choices across the session, indicating that neither strain was displaying any satiety effects in their choice behavior. And, both strains recaptured baseline between the delay and magnitude tasks indicating that there were no carry-over effects between tasks. An examination of the AUC and slope revealed a near-significant trend towards lower AUC (bottom panel of Figure 3) values in the magnitude task but there were no indications of any slope differences (bottom panel of Figures 4). Therefore, the LEW presented a small impulsive bias, but normal reward sensitivity in comparison to the WIS in the reward magnitude task (Figure 6). These results suggest that the LEW rats may possess some subtle deficits in reward magnitude valuation that could result in increases in impulsive choice behavior. Further research will be needed to determine the potential source of these deficits.

In the delay to reward manipulation, the preference for the LL reward increased when the delay to the SS reward increased in separate phases (Figure 2). During baseline, when the delay to reward in the SS was $10 \mathrm{~s}$, there was no difference in the choices of LL reward for WIS and LEW rats; however, when the delay to SS reward increased to $15 \mathrm{~s}$ and then to $20 \mathrm{~s}$, the LEW strain chose fewer LL rewards than the WIS controls. This was apparent in both the original choice measure and in the log-odds transformation. Both strains recaptured baseline between the magnitude and delay tasks indicating no significant carry-over effects. There was a general trend towards impulsive bias in the AUC values and shallower slopes in the delay task (Figures 3 and 4). 
The results confirm previous findings that LEW rats made significantly more SS choices in a delay discounting task when the delay to reward was manipulated [89, 91-94], and extends this finding to comparisons with their source strain, WIS. Stein et al. [90] recently reported steeper discounting functions in Lewis vs. Fischer 344 rats using an adjusting amount procedure delivered at different delays, but the delays were adjusted following stability (coupled with assessments of adjusting amounts for each delay). When delays were adjusted pseudorandomly for each session, then there were no differences in choice behavior between the two strains [see also 106]. It is difficult to determine the relative impact of the adjusting amount vs. the changes in delay that were delivered in Stein et al.'s procedure in producing the differences between the strains. It may be preferable to assess discounting separately for delay and amount adjustments as was the case in the current study to disentangle the separate influences of these two variables [see also 78, 95, 107]. From the present results it appears that the LEW rats possess more robust deficits in delay processing than in magnitude processing, but that impairments in both processes may be playing a role in their impulsive choice behavior.

An additional finding of interest was the observation that the LEW rats displayed decreased LL choices over the session in the delay manipulation only (Figure 5). This was in contrast to the WIS (and also SHR and WKY) strains that showed increased LL choices over the session. The decrease in LL choices was unlikely due to satiety effects on choice behavior because the rats were receiving the smallest LL magnitude ( 2 pellets) in the delay task. In addition, the LEW rats displayed an increase in LL choices over the session in the magnitude task, where satiety effects should have been more likely to occur. It is possible that the decrease in LL choices over time may reflect delay aversion in the LEW rats, which may lend further support to their consideration as an animal model of ADHD. Delay aversion has been reported 
as an important factor in impulsive choice behavior in ADHD patients [48, 49]. It is possible that delay aversion contributed to the shallower discounting function in the delay manipulation. Further research examining this factor will be required to parse out the impact of delay aversion vs. steeper discounting in affecting impulsive choice behavior in the LEW strain.

An additional possible source of the LEW deficits in the delay manipulation may be due to deficits in perceiving the change in delay or in discriminating between the successive SS delays. Interestingly, in addition to deficits in duration discrimination $[58,60]$, it has been proposed that ADHD involves deficits in detection of prediction errors with regard to the timing, nature, or frequency of changing events [108], which could provide an alternative explanation for the pattern of results in the LEW strain if they could not detect the prediction error when the SS delay was altered. Alternatively, the weak modulation of choice behavior with changes in the experimental contingencies may be the result of deficits in attention [80, 87]. Further research will be needed to determine the source of the deficits in the LEW strain. Additional research is also required to establish on which other indices of ADHD (e.g., executive functioning, working memory impairments, response variability) LEW and WIS rats may differ.

In relation to the LEW strain as a possible model of ADHD, this strain does show some interesting behavioral and neurobiological effects that may be relevant to their consideration. The LEW strain (relative to Fischer 344) is more likely to self-administer a variety of drugs including alcohol, cocaine, heroin, morphine, and nicotine [109-113]. In addition, GarciaLecumberri et al. [92] demonstrated that the LEW rats self-administered more morphine and also made more SS choices in an impulsive choice task, indicating that impulsive choice in the LEW strain may be a pre-existing trait that increases susceptibility to drug use. Given that ADHD is associated with increased impulsive choice and susceptibility to drug addiction [30,31], this 
suggests that the LEW strain could be a potential model for this aspect of ADHD. In addition, the LEW strain have lower levels of dopamine (DA) and dopamine transporters (DAT) in the nucleus accumbens and striatum, lower levels of serotonin (5-HT) in the nucleus accumbens, and decreased 5-HT1A receptors in frontal cortex and hippocampus [see 93]. Reductions in DA and 5-HT in these brain areas are also implicated in disordered impulsive choice and reward anticipation in ADHD [10, 11, 15, 71-73, 114].

Although it may be tempting to consider the LEW strain as a possible model for ADHD (at least with regard to impulsive choice), it is critical to note that the WIS and the LEW strains demonstrated high inter-individual variability in their impulsive choice behavior. Although a review of the literature did not reveal any published reports of subpopulations within the LEW strain, the present results suggested that this may be a possibility given the high inter-individual differences observed.

4.3. Overall summary and conclusion. The present research did not find support for considering the SHR strain as a model of ADHD. SHRs did not make significantly more impulsive choices than WKY controls and they did not show any deficits in adjusting to changes in magnitude or delay in comparison to WKY in an impulsive choice task. Individual differences among rats within a strain accounted for a significant proportion of the total variance in choice behavior and contributed substantially more variance than the strain of the rat. The LEW rats did display deficits in choice behavior, with robust effects on the delay task and more modest effects on the magnitude task, suggesting their consideration as a possible model of at least some aspects of ADHD, but further research will be needed to determine whether they are a viable model. A major concerning factor revealed in the present study is the high interindividual differences in all strains of rats, which raises issues for using the SHR and LEW 
strains as animal models because they may not be sufficiently homogeneous with respect to impulsive choice behavior to be considered as viable models for disordered impulsive choice. In a sense, this should not be entirely surprising given that neither of these strains have been specifically bred for serving as models of ADHD.

Measures of hyperactivity, impulsivity and inattention are normally distributed in the general population, and ADHD is regarded as the extreme end of these quantitative traits [51]. There are two main approaches to examining impulsive choice behavior in ADHD samples that could be applied favorably to animal model research. One approach is to examine clinical populations in comparison to non-clinical controls. To develop an animal model of clinical ADHD, one should aim to isolate important phenotypes by cross-breeding individuals that show ADHD-like characteristics (based on sampling from a normal outbred population), or by selective knock out of sets of relevant genes. Given that ADHD is a heterogeneous disorder with subtypes that may consist of clusters of related deficits, more than one animal model of ADHD will likely be needed [115]. To facilitate the identification and selection of the relevant phenotype, numerous behavioral correlates of the disease need to be measured within the same individual and clusters of related deficits identified. Future animal model development should aim to promote robust behavioral tasks with measurements designed to dissociate subtypes, or robust clusters of symptoms of ADHD, using methods such as those used in the present experiment. Research in clinical populations utilizing this approach can help guide development of appropriate animal models. A second approach is to utilize population samples and examine individual differences at the extremes, or across the entire population $[13,49,51,116]$. With regard to animal studies, attention should be paid to the heterogeneity of individuals within a strain [117], with examinations of individual variation along the bias vs. sensitivity space to 
determine factors that differentiate those individuals along quantitative dimensions that could serve as candidate endophenotypes [6]. Regardless of which approach is used, extending both the understanding of ADHD in human samples as well as developing animal models will be arduous, although necessary given the prevalence of ADHD and the importance of understanding the mechanisms that underlie impulsive behavior that is such an important factor in the disorder. 


\section{Acknowledgements}

The authors would like to acknowledge Angela Crumer for her help in the individual difference analysis and to Marina Vilardo for her help in running the study. This project formed the basis of a master's thesis submitted to Kansas State University by Ana Garcia, who was supported by Consejo Nacional de Ciencia y Tecnología (CONACYT). This research was also supported by a grant from National Institutes of Mental Health to Kansas State University (Grant number 5RO1MH085739). 


\section{References}

[1] Faraone SV, Sergeant J, Gillberg C, Biederman J. The worldwide prevalence of ADHD: is it an American condition? World Psychiatry. 2003;2:104-13.

[2] Froehlich TE, Lanphear BP, Epstein JN, Barbaresi WJ, Katusic SK, Kahn RS. Prevalence, recognition, and treatment of Attention-Deficit/Hyperactivity Disorder in a national sample of US children. Arch Pediatr Adolesc Med. 2007;161:857-64.

[3] Kessler RC, Adler L, Barkley R, Biederman J, Conners CK, Demler O, et al. The prevalence and correlates of adult ADHD in the United States: Results from the national comorbidity survey replication. Am J Psychiatry. 2006;163:716-23.

[4] Polanczyk G, Silva de Lima M, Horta BL, Biederman J, Rohde LA. The worldwide prevalence of ADHD: A systematic review and metaregression analysis. Am J Psychiatry. 2007; 164:942-8.

[5] APA. Diagnostic and statistical manual of mental disorders, IV. Washington, DC: American Psychological Association; 1994.

[6] Castellanos FX, Tannock R. Neuroscience of attention-deficit/hyperactivity disorder: The search for endophenotypes. Nature Reviews Neuroscience. 2002;3:617-28.

[7] Castellanos FX, Sonuga-Barke EJS, Milham MP, Tannock R. Characterizing cognition in ADHD: beyond executive dysfunction. Trends in Cognitive Sciences. 2006;10:117-23.

[8] Nigg JT. What causes ADHD? Understanding what goes wrong and why. New York: Guilford; 2006.

[9] Sonuga-Barke EJS, Wiersema JR, van der Meere JJ, Roeyers H. Context-dependent dynamic processes in attention deficit/hyperactivity disorder: differentiating common and unique effects of state regulation deficits and delay aversion. Neuropsychol Rev. 2010;20:86-102. 
[10] Sonuga-Barke EJS. Psychological heterogeneity in AD/HD-A dual pathway model of behaviour and cognition. Behav Brain Res. 2002;10:29-36.

[11] Sonuga-Barke EJS. The dual pathway model of AD/HD: An elaboration of neurodevelopmental characteristics. Neurosci Biobehav Rev. 2003;27:593-604.

[12] Sonuga-Barke EJS, Bitsakou P, Thompson M. Beyond the dual pathway model: Evidence for the dissociation of timing, inhibitory, and delay-related impairments in AttentionDeficit/Hyperactivity Disorder. J Am Acad Child Adolesc Psychiatry. 2010;49:345-55. [13] Wählstedt C, Thorell LB, Bohlin G. Heterogeneity in ADHD: Neuropsychological pathways, comorbidity and symptom domains. J Abnorm Child Psychol. 2009;37:551-64. [14] Evenden JL. Varieties of impulsivity. Psychopharmacology (Berl). 1999;146:348-61. [15] Winstanley CA, Eagle DM, Robbins TW. Behavioral models of impulsivity in relation to ADHD: Translation between clinical and preclinical studies. Clin Psychol Rev. 2006;26:379-95. [16] Mazur JE. Rats' choices between one and two delayed reinforcers. Learning \& Behavior. 2007;35:169-76.

[17] Ainslie GW. Impulse control in pigeons. Journal of the Experimental Analysis of Behavior. 1974;21:485-9.

[18] Logue AW. Research on self-control an integrating framework. Behavioural Brain Research. 1988;11:665-709.

[19] de Wit H. Impulsivity as a determinant and consequence of drug use: a review of underlying processes. Addiction Biology. 2008;14:22-31.

[20] MacKillop J, Amlung MT, Few LR, Ray LA, Sweet LH, Munafo MR. Delayed reward discounting and addictive behavior: a meta-analysis. Psychopharmacology (Berlin). 2011;216. 
[21] Reynolds B. A review of delay-discounting research with humans: Relations to drug use and gambling. Behav Pharmacol. 2006;17:651-67.

[22] Dixon MR, Jacobs EA, Sanders S. Contextual control of delay discounting by pathological gamblers. J Appl Behav Anal. 2006;39:413-22.

[23] Dixon MR, Marley J, Jacobs EA. Delay discounting by pathological gamblers. J Appl

Behav Anal. 2003;36:449-58.

[24] Petry NM, Casarella T. Excessive discounting of delayed rewards in substance abusers with gambling problems. Drug Alcohol Depend. 1999;56:25-32.

[25] Alessi SM, Petry NM. Pathological gambling severity is associated with impulsivity in a delay discounting procedure. Behavioral Processes. 2003;64:345-54.

[26] Angeletos G-M, Laibson D, Repetto A, Tobacman J, Weinberg S. The hyperbolic consumption model: Calibration, simulation, and empirical evaluation. J Econ Perspect. 2001;15:47-68.

[27] Krishnan-Sarin S, Reynolds B, Duhig AM, Smith A, Liss T, McFetridge A, et al. Behavioral impulsivity predicts treatment outcome in a smoking cessation program for adolescent smokers. Drug Alcohol Depend. 2007;88:79-82.

[28] Landes RD, Christensen DR, Bickel WK. Delay discounting decreases in those completing treatment for opioid dependence. Experimental and Clinical Psychopharmacology. 2012;20:3029.

[29] Yoon JH, Higgins ST, Heil SH, Sugarbaker RJ, Thomas CS, Badger GJ. Delay discounting predicts postpartum relapse to cigarette smoking among pregnant women. Experimental and Clinical Psychopharmacology. 2007;15:176-86. 
[30] Urcelay GP, Dalley JW. Linking ADHD, impulsivity, and drug abuse: A neuropsychological perspective. In: Stanford C, Tannock R, editors. Behavioral Neuroscience of Attention Deficit Hyperactivity Disorder and Its Treatment. Berlin: Springer-Verlag; 2012. [31] Verdejo-García A, Lawrence AJ, Clark L. Impulsivity as a vulnerability marker for substance-use disorders: review of findings from high-risk research, problem gamblers and genetic association studies. Neurosci Biobehav Rev. 2008;32:777-810.

[32] Barkley RA, Edwards G, Laneri M, Fletcher K, Metevia L. Executive functioning, temporal discounting, and sense of time in adolescents with attention deficit hyperactivity disorder (ADHD) and oppositional defiant disorder (ODD). J Abnorm Child Psychol. 2001;29:541-56. [33] Luman M, Oosterlaan J, Sergeant JA. The impact of reinforcement contingencies on AD/HD: A review and theoretical appraisal. Clin Psychol Rev. 2005;25:183-213. [34] Sonuga-Barke EJS, Taylor E, Sembi S, Smith J. Hyperactivity and delay aversion. I. The effect of delay on choice. Journal of Child Psychology and Psychiatry. 1992;33:387-98.

[35] Scheres A, Lee A, Sumiya M. Temporal reward discounting and ADHD: task and symptom specific effect. J Neural Transm. 2008;115:221-6.

[36] Kuntsi J, Oosterlaan J, Stevenson J. Psychological mechanisms in hyperactivity. I. Response inhibition deficit, working memory impairment, delay aversion, or something else? Journal of Child Psychology and Psychiatry. 2001;42:199-210.

[37] Schweitzer JB, Sulzer-Azaroff B. Self-control in boys with attention deficit hyperactivity disorder: Effects of added stimulation and time. Journal of Child Psychology and Psychiatry. 1995;36:671-86.

[38] Tripp G, Alsop B. Sensitivity to reward delay in children with attention deficit hyperactivity disorder (ADHD). Journal of Child Psychology and Psychiatry. 2001;42:691-8. 
[39] Neef NA, Bicard DF, Endo S. Assessment of impulsivity and the development of selfcontrol in students with attention deficit hyperactivity disorder. J Appl Behav Anal. 2001;34:397-408.

[40] Antrop I, Stock P, Verte' S, Wiersema JR, Baeyens D, Roeyers H. ADHD and delay aversion: The influence of nontemporal stimulation on choice for delayed rewards. Journal of Child Psychology and Psychiatry. 2006;47:1152-8.

[41] Neef NA, Marckel J, Ferreri SJ, Bicard DF, Endo S, Aman MG, et al. Behavioral assessment of impulsivity: A comparison of children with and without attention deficit hyperactivity disorder. J Appl Behav Anal. 2005;38:23-37.

[42] Hoerger ML, Mace FC. A computerized test of self-control predicts classroom behavior. J Appl Behav Anal. 2006;39:147-59.

[43] Bitsakou P, Psychogiou L, Thompson M, Sonuga-Barke EJS. Delay aversion in Attention Deficit/Hyperactivity Disorder: an empirical investigation of the broader phenotype. Neuropsychologia. 2009;47:446-56.

[44] Solanto MV, Abikoff H, Sonuga-Barke E, Schachar R, Logan GD, Wigal T, et al. The ecological validity of delay aversion and response inhibition as measures of impulsivity in AD/HD: A supplement to the NIMH multimodal treatment study of AD/HD. J Abnorm Child Psychol. 2001;29:215-28.

[45] Scheres A, Dijkstra M, Ainslie E, Balkan J, Reynolds B, Sonuga-Barke E, et al. Temporal and probabilitistic discounting of rewards in children and adolescents: Effects of age and ADHD symptoms. Neuropsychologia. 2006;44:2092-103. 
[46] Scheres A, Tontsch C, Theony AL, Kaczkurkin A. Temporal reward discounting in Attention-Deficit/Hyperactivity Disorder: The contribution of symptom domains, reward magnitude, and session length. Biol Psychiatry. 2010;67:641-8.

[47] Wilson VB, Mitchell SH, Musser ED, Schmitt CF, Nigg JT. Delay discounting of reward in ADHD: application in young children. Journal of Child Psychology and Psychiatry. 2011;52:256-64.

[48] Dalen L, Sonuga-Barke EJS, Hall M, Remington B. Inhibitory deficits, delay aversion and preschool AD/HD: Implications for the dual pathway model. Neural Plas. 2004;11:1-11.

[49] Thorell LB. Do delay aversion and executive function deficits make distinct contributions to the functional impact of ADHD symptoms? A study of early academic skill deficits. Journal of Child Psychology and Psychiatry. 2007;48:1061-70.

[50] Marco R, Miranda A, Melia A, MUller U, Butler L, Gabriels I, et al. Delay and reward choice in ADHD: An experimental test of the role of delay aversion. Neuropsychology. 2009;23:367-80.

[51] Paloyelis Y, Asherson P, Kuntsi J. Are ADHD symptoms associated with delay aversion or choice impulsivity? A general population study. J Am Acad Child Adolesc Psychiatry. 2009;48:837-46.

[52] Toplak ME, Dockstader C, Tannock R. Temporal information processing in ADHD:

Findings to date and new methods. J Neurosci Methods. 2006;151:15-29.

[53] Smith A, Taylor E, Rogers JW, Newman S, Rubia K. Evidence for a pure time perception deficit in children with ADHD. Journal of Child Psychology and Psychiatry. 2002;43:529-42. [54] Luman M, Oosterlaan J, Sergeant JA. Modulation of response timing in ADHD, Effects of reinforcement valence and magnitude. J Abnorm Child Psychol. 2008;36:445-56. 
[55] Baldwin RL, Chelonis JJ, Flake RA, Edwards MC, Feild CR, Meaux JB, et al. Effect of methylphenidate on time perception in children with Attention-Deficit/Hyperactivity Disorder. Experimental and Clinical Psychopharmacology. 2004;12:57-64.

[56] Barkley RA, Koplowitz S, Anderson T, McMurray MB. Sense of time in children with ADHD: effects of duration, distraction, and stimulant medication. J Int Neuropsychol Soc. 1997;3:359-69.

[57] Cappella B, Gentile JR, Juliano DB. Time estimation by hyperactive and normal children. Percept Mot Skills. 1977;44:787-90.

[58] Huang J, Yang B, Zou X, Jing J, Pen G, McAlonan GM, et al. Temporal processing impairment in children with attention-deficithyperactivity disorder. Res Dev Disabil. 2012;33:538-48.

[59] Kerns KA, McInerney RJ, Wilde NJ. Time reproduction, working memory, and behavioral inhibition in children with ADHD. Child Neuropsychology. 2001;7:21-31.

[60] Yang B, Chan RCK, Zou X, Jing J, Mai J, Li J. Time perception deficit in children with ADHD. Brain Res. 2007;1170:90-6.

[61] Johansen EB, Aase H, Meyer A, Sagvolden T. Attention-deficit/hyperactivity disorder (ADHD) behaviour explained by dysfunctioning reinforcement and extinction processes. Behav Brain Res. 2002;130:37-45.

[62] Meck WH. Neuroanatomical localization of an internal clock: a functional link between mesolimbic, nigrostriatal, and mesocortical dopaminergic systems. Brain Res. 2006;1109:93107.

[63] Coull JT, Cheng R-K, Meck WH. Neuroanatomical and neurochemical substrates of timing. Neuropsychopharmacology. 2011;36:3-25. 
[64] Holroyd CB, Baker TE, Kerns KA, Maller U. Electrophysiological evidence of atypical motivation and reward processing in children with attention-deficit hyperactivity disorder. Neuropsychologia. 2008;46:2234-42.

[65] Scheres A, Milham MP, Knutson B, Castellanos FX. Ventral striatal hyporesponsiveness during reward anticipation in Attention-Deficit/Hyperactivity Disorder. Biol Psychiatry. $2007 ; 61: 720-4$.

[66] Johansen EB, Killeen PR, Russell VA, Tripp G, Wickens JR, Tannock R, et al. Origins of altered reinforcement effects in ADHD. Behavioral and Brain Functions. 2009;5:1-15. [67] Volkow ND, Wang G-J, Kollins SH, Wigal TL, Newcorn JH, Telang F, et al. Evaluating dopamine reward pathway in ADHD: Clinical implications. JAMA. 2009;301:1084-91. [68] Volkow ND, Wang G-J, Newcorn JH, Kollins SH, Wigal TL, Telang F, et al. Motivation deficit in ADHD is associated with dysfunction of the dopamine reward pathway. Mol Psychiatry. 2010;15:1147-54.

[69] Volkow ND, Wang G-J, Newcorn JN, Telang F, Solanto MV, Fowler JS, et al. Depressed dopamine activity in caudate and preliminary evidence of limbic involvement in adults with Attention-Deficit/Hyperactivity Disorder. Arch Gen Psychiatry. 2007;64:932-94. [70] Plichta MM, Vasic N, Wolf RC, Lesch KP, Brummer D, Jacob C, et al. Neural hyporesponsiveness and hyperresponsiveness during immediate and delayed reward processing in adult Attention-Deficit/Hyperactivity Disorder. Biol Psychiatry. 2009;65:7-14.

[71] Ströhle A, Stoy M, Wrase J, Schwarzer S, Schlagenhauf F, Huss M, et al. Reward anticipation and outcomes in adult males with attention-deficit/hyperactivity disorder. Neuroimage. 2008;39:966-72. 
[72] Carmona S, Hoekzema EA, Ramos-Quiroga JA, Richarte V, Canals C, Bosch R, et al. Response inhibition and reward anticipation in medication-nave adults with AttentionDeficit/Hyperactivity Disorder: A within-subject case-control neuroimaging study. Hum Brain Mapp. 2011;33:2350-61.

[73] Carmona S, Proal E, Hoekzema EA, Gispert J-D, Picado M, Moreno I, et al. Ventro-striatal reductions underpin symptoms of hyperactivity and impulsivity in AttentionDeficit/Hyperactivity Disorder. Biol Psychiatry. 2009;66:972-7.

[74] Belin D, Jonkman S, Dickinson A, Robbins TW, Everitt BJ. Parallel and interactive learning processes within the basal ganglia: Relevance for the understanding of addiction. Behav Brain Res. 2009;199:89-102.

[75] Olausson P, Jentsch JD, Tronson N, Neve RL, Nestler EJ, Taylor JR. $\Delta$ FoSB in the nucleus accumbens regulates food-reinforced instrumental behavior and motivation. The Journal of Neuroscience. 2006;26:9196-204.

[76] Zhang M, Balmadrid C, Kelley A. Nucleus accumbens opiod, GABAergic and dopaminergic modualtion of palatable food motivation: Contrasting effects revealed by a progressive ratio study in the rat. Behav Neurosci. 2003;117:202-11.

[77] Robbins TW, Everitt BJ. Neurobehavioural mechanisms of reward and motivation. Curr Opin Neurobiol. 1996;6:228 - 36.

[78] Galtress T, Kirkpatrick K. The role of the nucleus accumbens core in impulsive choice, timing, and reward processing. Behav Neurosci. 2010;124:26-43.

[79] Davids E, Zhang K, Tarazi FI, Baldessarini RJ. Animal models of attention-deficit hyperactivity disorder. Brain Research - Brain Research Reviews. 2003;42:1-21. 
[80] Sagvolden T. Behavioral validation of the spontaneously hypertensive rat (SHR) as an animal model of attention-deficit/hyperactivity disorder (AD/HD). Neurosci Biobehav Rev. $2000 ; 24: 31-9$

[81] Sagvolden T, Johansen EB. Rat models of ADHD. In: Stanford C, Tannock R, editors. Behavioral Neuroscience of Attention Deficit Hyperactivity Disorder and Its Treatment. Berlin: Springer-Verlag; 2012.

[82] Hand DJ, Fox AT, Reilly MP. Differential effects of d-amphetamine on impulsive choice in spontaneously hypertensive and Wistar-Kyoto rats. Behav Pharmacol. 2009;20:549-53.

[83] Fox AT, Hand DJ, Reilly MP. Impulsive choice in a rodent model of attentiondeficit/hyperactivity disorder. Behav Brain Res. 2008;187:146-52.

[84] Bizot J-C, Chenault N, Houzé B, Herpin A, David S, Pothion S, et al. Methylphenidate reduces impulsive behaviour in juvenile Wistar rats, but not in adult Wistar, SHR and WKY rats. Psychopharmacology (Berl). 2007;193:215-23.

[85] Wooters TE, Bardo MT. Methylphenidate and fluphenazine, but not amphetamine, differentially affect impulsive choice in Spontaneously Hypertensive, Wistar-Kyoto and Sprague-Dawley rats. Brain Res. 2011;1396:45-53.

[86] Sutherland KR, Alsop B, McNaughton N, Hyland BI, Tripp G, Wickens JR. Sensitivity to delay of reinforcement in two animal models of attention deficit hyperactivity disorder (ADHD). Behav Brain Res. 2009;205:372-6.

[87] Adriani W, Caprioli A, Granstrem O, Carli M, Laviola G. The spontaneously hypertensiverat as an animal model of ADHD: evidence for impulsive and nonimpulsive subpopulations. Neuroscience Biobehavioral Reviews 2003;27: 639-51. 
[88] Orduña V, Garcia A, Hong E. Choice behavior in spontaneously hypertensive rats: Variable vs. fixed schedules of reinforcement. Behavioural Processes. 2010;84:465-9.

[89] Madden GJ, Smith NG, Brewer AT, Pinkston JW, Johnson PS. Steady-state assessment of impulsive choice in Lewis and Fischer 344 rats: Between-condition delay manipulations. J Exp Anal Behav. 2008;90:333-44.

[90] Stein JS, Pinkston JW, Brewer AT, Francisco MT, Madden GJ. Delay discounting in Lewis and Fischer 344 rats: Steady-state and rapid-determination adjusting-amount procedures. J Exp Anal Behav. 2012;97:305-21.

[91] Anderson KG, Diller JW. Effects of acute and repeated nicotine administration on delay discounting in Lewis and Fischer 344 rats. Behav Pharmacol. 2010;21:754-64.

[92] García-Lecumberri C, Torres I, Martín S, Crespo JA, Miguéns M, Nicanor C, et al. Strain differences in the dose-response relationship for morphine self-administration and impulsive choice between Lewis and Fischer 344 rats. J Psychopharmacol (Oxf). 2010;25:783-91.

[93] Huskinson SL, Krebs CA, Anderson KG. Strain differences in delay discounting between Lewis and Fischer 344 rats at baseline and following acute and chronic administration of damphetamine. Pharmacol, Biochem Behav. 2012;101:403-16.

[94] Anderson KG, Woolverton WL. Effects of clomipramine on self-control choice in Lewis and Fischer 344 rats. Pharmacol, Biochem Behav. 2005;80:387-93.

[95] Roesch MR, Takahashi Y, Gugsa N, Bissonette GB, Schoenbaum G. Previous cocaine exposure makes rats hypersensitive to both delay and reward magnitude. The Journal of Neuroscience. 2007;27:245-50.

[96] Roberts S. Isolation of an internal clock. J Exp Psychol Anim Behav Process. 1981;7:24268. 
[97] Odum AL. Delay discounting: I'm a k, your're a k. J Exp Anal Behav. 2011;96:427-39.

[98] Garcia Aguirre AI. Timing, reward processing and choice behavior in four strains of rats with different levels of impulsivity. Manhattan, KS: Kansas State University; 2011.

[99] Olejnik S, Algina J. Generalized eta and omega squared statistics: Measures of effect size for some common research designs. Psychological Methods. 2003;8:434-47.

[100] Bakeman R. Recommended effect size statistics for repeated measures designs. Behavior Research Methods. 2005;37:379-84.

[101] Madden GJ, Francisco MT, Brewer AT, Stein JS. Delay discounting and gambling. Behavioural Processes. 2011;87:43-9.

[102] Myerson J, Green L, Warusawitharana M. Area under the curve as a measure of discounting. J Exp Anal Behav. 2001;76:235-43.

[103] Sagvolden T, DasBanerjee T, Zhang-James Y, Middleton F, Faraone S. Behavioral and genetic evidence for a novel animal model of Attention-Deficit/Hyperactivity Disorder predominately innatentive subtype. Behavioral and Brain Functions. 2008;4:1-11.

[104] Sagvolden T, Johansen EB, Wøien G, Walaas SI, Storm-Mathisen J, Bergersen LH, et al. The spontaneously hypertensive rat model of ADHD - The importance of selecting the appropriate reference strain. Neuropharmacology. 2009;57:619-26.

[105] Nabika T, Nara Y, Ikeda K, Endo J, Yamori Y. Genetic heterogeneity of the spontaneously hypertensive rat. Hypertension (Dallas). 1991;18:12-6.

[106] Wilhelm CJ, Mitchell SH. Strain differences in delay discounting using inbred rats. Genes, Brain and Behavior. 2009;8:426-34. 
[107] Ho MY, Mobini S, Chiang TJ, Bradshaw CM, Szabadi E. Theory and method in the quantitative analysis of "impulsive choice" behavior: implications for psychopharmacology. Psychopharmacology (Berlin). 1999;146:362-72.

[108] Nigg JT, Casey BJ. An integrative theory of attention-deficit/hyperactivity disorder based on the cognitive and affective neurosciences. Dev Psychopathol. 2005;17:785-806. [109] Brower VG, Fu Y, Matta SG, Sharp BM. Rat strain differences in nicotine selfadministration using an unlimited access paradigm. Brain Res. 2002;930:12-20. [110] Kosten TA, Miserendino MJ, Haile CN, DeCaprio JL, Jatlow PI, Nestler EJ. Acquisition and maintenance of intravenous cocaine self-administration in Lewis and Fischer inbred rat strains. Brain Res. 1997;778:418-29.

[111] Suzuki T, George FR, Meisch RA. Differential establishment and maintenance of oral ethanol reinforced behavior in Lewis and Fischer 344 inbred rat strains. J Pharmacol Exp Ther. 1988;245:164-70.

[112] Picetti R, Caccavo JA, Ho A, Kreek MJ. Dose escalation and dose preference in extendedaccess heroin self-administration in Lewis and Fischer rats. Psychopharmacology (Berlin). 2012;220:163-72.

[113] Martin S, Manzanares J, Corchero J, Garcia-Lecumberri C, Crespo JA, Fuentes JA, et al. Differential basal proenkaphalin gene expression in dorsal striatum and nucleus accumbens, and vulnerability to morphine self-administration in Fischer 344 and Lewis rats. Brain Res. $1999 ; 821: 350-5$.

[114] Tripp G, Wickens JR. Research Review: Dopamine transfer deficit: a neurobiological theory of altered reinforcement mechanisms in ADHD. Journal of Child Psychology and Psychiatry. 2008;49:691-704. 
[115] Wickens JR, Hyland BI, Tripp G. Animal models to guide clinical drug development in ADHD: lost in translation? Br J Pharmacol. 2011;164:1107-28.

[116] Fair DA, Bathula D, Nikolas MA, Nigg JT. Distinct neuropsychological subgroups in typically developing youth inform heterogeneity in children with ADHD. Proceedings of the National Academy of Sciences. 2012;109:6769-74.

[117] Galtress T, Garcia A, Kirkpatrick K. Individual differences in impulsive choice and timing in rats. J Exp Anal Behav. 2012;98:65-87. 


\section{Figure Captions}

Figure 1. The percentage of choices made to the LL lever for the WKY and SHR strains. The left column shows the data for the LL magnitude manipulation, while right column shows the data in the SS delay manipulation. The top row displays the group means and the middle and bottom rows show the box plots for each individual strain. The different phases in the LL reward magnitude manipulation are labeled according to the number of pellets received on the LL lever in each phase. In the SS delay manipulation, the different phases are labeled according to the delay to the receipt of the SS reward in each phase. The LL delay was always $30 \mathrm{~s}$ and the SS reward was always 1 pellet.

Figure 2. Percentage of choices made to the LL lever for the WIS and LEW strains during the LL magnitude (left column) and SS delay (right column) manipulations. The top row displays the group means and the middle and bottom rows show the box plots for each individual strain. Figure 3. Mean \pm standard error of the mean (SEM) of the area under the curve (AUC) for the WKY vs. SHR (top panel) and WIS vs. LEW (bottom panel) strains in the LL magnitude (M; filled symbols) and SS delay (D; open symbols) manipulations.

Figure 4. Mean \pm SEM of the slope of the discounting function for the WKY vs. SHR (top panel) and WIS vs. LEW (bottom panel) strains in the LL magnitude (M; filled symbols) and SS delay (D; open symbols) manipulations..

Figure 5. Mean $( \pm$ SEM) of the percentage of LL choices during the first vs. second half of the session for the WKY vs. SHR (top panel) and WIS vs. LEW (bottom panel) strains in the magnitude (M; filled symbols) and delay (D; open symbols) manipulations. 
Figure 6. Scatter plot of the AUC vs. slope for the LL magnitude (top panel) and SS delay (bottom panel) tasks comparing all four strains. The horizontal axis shows different levels of self-control; AUC values greater than 0.5 indicate more self-controlled choices (preference of LL). The vertical axis displays different levels of sensitivity to the magnitude or delay changes; higher values indicate greater sensitivity to change. Each quartile represents different patterns of performance where the upper right quartile would contain the ideal performance, which indicates generalized self-control bias coupled with good sensitivity to change, and the lower left quartile is the least ideal pattern, which indicates generalized impulsive bias coupled with poor sensitivity. 